\title{
Membranes and organelles
}

\section{Editorial overview Gillian Griffiths and Judith Klumperman}

\author{
Current Opinion in Cell Biology 2004, 16:341-342 \\ Available online 20th June 2004 \\ $0955-0674 / \$$ - see front matter \\ ๑ 2004 Published by Elsevier Ltd. \\ DOI 10.1016/j.ceb.2004.06.016
}

\section{Gillian Griffiths}

Sir William Dunn School of Pathology, South Parks Rd, Oxford OX1 3RE, UK

Gillian Griffiths is the Professor of Experimental Pathology at the Sir William Dunn School of Pathology, Oxford University, and a Wellcome Trust fellow. Her group studies secretory lysosomes and their sorting mechanisms.

\section{Judith Klumperman}

Cell Microscopy Center, Dept of Cell Biology, AZU RM G02.525, Heidelberglaan 100, 3584CX Utrecht, The Netherlands

Judith Klumperman is the Head of Cell Microscopy and Professor of Cell Biology at the University Medical Center, Utrecht. Her overall research interest is intracellular transport.

\section{Abbreviations \\ ER endoplasmic reticulum}

Compartmentalisation by intracellular membranes provides the basis for many different types of cell specialisations, which allow cells to execute an enormous number of different functions within the body. At the same time they lay down a complicated pathway within the cell for proteins to be sorted correctly to their destinations. However, within cells, both proteins and organelles are sorted with incredible accuracy and specificity. Our understanding of how this is accomplished is increasing as the mechanisms governing membrane trafficking are unravelled. This issue includes reviews on some of the current areas involving proteins travelling through eukaryotic cells.

Newly synthesised proteins are rigorously screened for mutation and misfolding as they are synthesised in the endoplasmic reticulum (ER). A number of different chaperones fulfil this role and act in concert to ensure that only correctly folded proteins are allowed to proceed past this point. Mis-folded proteins are retained and targeted for ER-associated degradation. Kleizen and Braakman address the question of how the chaperones work together and describe recent work identifying the co-factors required to distinguish between proteins destined for exit versus retention and degradation. This points to decisions being made at the ER exit site, a topic discussed by Watanabe and Riezman. In addition to COPII function, an increasing number of different mechanisms appear to contribute to cargo sorting in the ER, including lipid rafts, tethering factors and SNARE proteins. Interestingly, an emerging additional level of regulation of ER export is provided by the recent identification of subtypes of carriers, mediating transport of specific cargoes.

Post-translational modification of proteins and lipids required for further sorting and interactions occurs in the Golgi, which provides an assembly line of glycosyltransferases. The latest advances in the fine-tuning of glycan biosynthesis are described by de Graffenried and Bertozzi. This review highlights the need for specific associations between glycosyltransferases to establish their activation as well as their localisation within the Golgi. Remarkably, despite the dynamic turnover of the Golgi cisternae, both the proteins and lipids within the Golgi are maintained at a steady state. A number of Golgi-associated proteins now appear to play a role in obtaining Golgi homeostasis. The review by Altan-Bonnet, Sougrat and LippincottSchwartz provides an intriguing model of how these proteins in a contingent sequence of events may contribute to the balance between Golgi structurefunction and Golgi dynamics.

The last few years have seen an enormous increase in interest in the role of lipids in membrane trafficking. It is now clear that lipids are much more than 
passive containers for protein transport and play an active role in membrane trafficking. van Meer and Sprong review the various roles for lipids in facilitating membrane fusion and fission as well as in protein sorting and the formation of lipid rafts. With the emergence of lipids as receptors for viruses and as motifs in immune recognition, the question of how and where lipids are themselves localised and regulated is now emerging as an important area in membrane trafficking. One area where lipid modification is now well established is the process of endocytosis. McMahon and Mills provide a comprehensive overview on the similarities and differences between mechanisms which move proteins and lipids from one membrane system to another via vesicles and/or tubules, and describe the role of coat proteins during membrane transport. Especially, they highlight common structural features between the motif-based cargo recognition by COP and clathrin adaptor coats, exemplifying the commonalities between pre- and post-Golgi, secretory and endocytic transport. The review by Sorkin also discusses the role of coats in transport, but focuses on the specific sorting motifs used to recruit the AP-2 adaptor complex and cargo to clathrin-coated vesicles. The last few years have seen an explosion in the number of proteins that can act as clathrin adaptor proteins, including arrestins and mono-ubiquitin binding proteins such as Hrs, Epsin and Eps15. Some of these components appear to act in the formation of clathrin-coated vesicles in an AP-2 independent manner. A striking implication of these findings is that, just as seems to be the case for export from the ER, there may be a cargo-driven variability in the type of clathrin-coated vesicle used to enter the cell. Intriguingly the endocytic pathway is now fast emerging as an important area for signalling. Miaczynska, Pelkmans and Zerial describe a new role for endosomes: they are important organelles for signalling rather than simply a 'sink' used to down-regulate signalling proteins from the plasma membrane. In this way signal transduction can be regulated both spatially and temporally along the endocytic pathway, providing a novel mechanism of 'fine-tuning', a reiterating theme that emerges from a number of reviews in this issue.

Yet another novel role for endosomes is emerging from work in Drosophila, reviewed by Dudu, Pantazis and González-Gaitán. They describe the intriguing role of endosomes in cell-cell communication of morphogens involved in embryonic development. This is not entirely without precedent as 'exosomes' are membrane vesicles released from endocytic organelles that are able to provide signals between cells of the immune system. Février and Raposo describe the current state of knowledge of exosome function both in vitro and in vivo and the mechanisms that govern their release and biological effects. The finding that HIV buds into the multivesicular bodies from which exosomes are released suggests that retroviruses may use this pathway to spread from one cell to another in a manner perhaps analogous to the spread of Drosophila morphogen gradients.

Signalling and endocytosis also play an undeniably important role in phagocytic cells. Niedergang and Chavrier review recent findings on how signalling leads to the recruitment of internal membranes from endocytic compartments to the plasma membrane at the site of phagocytosis, a process called focal exocytosis, and subsequent formation of the phagosome used to engulf pathogens. Focal exocytosis involves SNARE proteins in every step of membrane fusion along the membrane trafficking pathway through the cell. The recent structural and functional studies on how SNARE complexes lead to membrane fusion are reviewed by Söllner. These studies reveal remarkable similarities between viral and intracellular fusion and highlight the role of synaptotagmins in providing calcium-regulated secretion via the SNARE complexes.

Much of the work on membrane trafficking over the past 25 years has focussed on understanding how polarised epithelial cells are able to sort proteins and lipids to either the apical or the basolateral surfaces. The review by Rodriguez-Boulan, Müsch and Le Bivic provides an inclusive overview of all the advances described in the context of polarised epithelial cells. An important factor in establishing cell polarity is interactions with the cytoskeleton, which are used by both vesicles and tubules to move throughout the cell. In neuronal cells the distances travelled on the microtubule cytoskeleton can be significant along the length of axons. The motor proteins used in these processes are discussed by Guzik and Goldstein. This review covers rapid advances in the mechanics of the different motors as well as the insights provided by a number of diseases arising from defects in motor proteins. Studies of diseases have also provided significant insights into the roles of various Rab proteins. Seabra and Wasmeier describe new findings on the many different mechanisms, which control localisation of Rab proteins with incredible specificity to particular organelles. They discuss the diverse roles of the Rab proteins through recognition of a wide range of effectors and the finetuning of these functions via mechanisms that regulate Rab binding. As with coat proteins, the interesting possibility is raised that Rabs can be recruited to the outside of vesicles by internal cargo.

An area in which disease studies have proven a remarkably successful trigger for identifying a number of novel protein complexes is the biogenesis of lysosomes. BLOCS (biogenesis of lysosome related organelle complexes) have been identified from studies of HermanskyPudlak syndrome, a disease characterised by dysfunction of lysosomes and lysosome-related organelles in platelets and melanocytes. Dell'Angelica provides a comprehensive and fascinating review on these novel complexes, which are found ubiquitously in higher eukaryotes. 